NSF-ITP-91-01

McGill/92-01

\title{
From polymers to quantum gravity: triple-scaling in rectangular random matrix models
}

\author{
Robert C. Myers ${ }^{1}$ \\ Physics Department, McGill University \\ Ernest Rutherford Building \\ Montréal, Québec, CANADA H3A 2T8 \\ Vipul Periwal ${ }^{2}$ \\ Institute for Advanced Study \\ School of Natural Science \\ Princeton, New Jersey 08540-4920
}

\begin{abstract}
Rectangular $N \times M$ matrix models can be solved in several qualitatively distinct large $N$ limits, since two independent parameters govern the size of the matrix. Regarded as models of random surfaces, these matrix models interpolate between branched polymer behaviour and two-dimensional quantum gravity. We solve such models in a 'triplescaling' regime in this paper, with $N$ and $M$ becoming large independently. A correspondence between phase transitions and singularities of mappings from $\mathbf{R}^{2}$ to $\mathbf{R}^{2}$ is indicated. At different critical points, the scaling behavior is determined by: i) two decoupled ordinary differential equations; ii) an ordinary differential equation and a finite difference equation; or iii) two coupled partial differential equations. The Painlevé II equation arises (in conjunction with a difference equation) at a point associated with branched polymers. For critical points described by partial differential equations, there are dual weak-coupling/strong-coupling expansions. It is conjectured that the new physics is related to microscopic topology fluctuations.
\end{abstract}

1 rcm@physics.mcgill.ca

2 vipul@guinness.ias.edu. Also at Institute for Theoretical Physics, University of California, Santa Barbara, CA 93106-4030 


\section{Introduction}

Advances have been made in the study of noncritical string theory with the discovery of the double-scaling limit[1] in random matrix models. The double-scaling limit correlates the approach of the couplings in the matrix model to certain critical values with the large- $N$ limit. We give herein the solution of rectangular $N \times M$ matrix models in a 'triple-scaling' regime $-N$ and $M$ are taken to infinity independently in correlation with the approach to critical matrix couplings. The surface interpretation of these models in certain limits has been described in previous work $[2,3]$. The interest in these models is two-fold: (a) they interpolate between the rather different physics of random surfaces, and branched polymers, and (b) they exhibit critical behaviour involving two scaling variables. We list explicitly the novel results/techniques contained in the present work:

1. We find phase transitions in correspondence to singularities of mappings from $\mathbf{R}^{2}$ to $\mathbf{R}^{2}$.

2. We use recursion relations relating polynomials for different measures on the half-line. The significance of this lies in the fact that the derivative terms in the differential equations that describe critical behaviour can arise from either recursion relations relating different values of $N$ or $M$ (or linear combinations of these two parameters).

3. The resulting scaling behavior may be governed by: (i) two decoupled ordinary differential equations; (ii) an ordinary differential equation and a finite difference equation; or (iii) two coupled partial differential equations.

4. In case (iii) above, the equations admit weak-coupling solutions in either variable, which are simultaneously strong-coupling expansions in the other variable.

Since the results and techniques given in the present paper are largely unrelated to the contents of Ref.'s 2,3, it can be read independently of our previous work. To put it in context, we mention that in work with Anderson[2,3], we solved rectangular $N \times M$ matrix models in two distinct double-scaling limits, with either $M$ (limit I) ${ }^{\dagger}$ or $N-M$ (limit II) $)^{\ddagger}$ held fixed as $N$ diverges. The limit I models describe dynamically branching polymers[4,3], while the limit II models yield two-dimensional random surfaces[6,2]. In Ref. 7, one of us (R.C.M.) solved coupled vector models in a triple-scaling limit, which has some features in common with the rectangular matrices solved in the present work. We wish to draw attention to the work of G.M. Cicuta and collaborators[8], who solved rectangular matrix models in the planar limit several years ago*, and to the work of J. Minahan, who has solved matrix models for surfaces with boundaries $[9,10]$ - some of the equations we shall obtain in the following also arise in his studies (see sect. 4).

The organization of this paper is as follows:

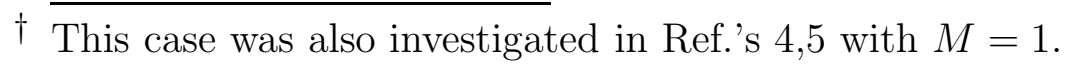

$\ddagger$ Ref.'s 6,5 analyzed this case with $N-M=0$.

* We regret that we were unaware of this work when Ref.'s 2,3 were published. 
- Sect. 1 contains some notation, the Jacobian of interest, and a list of properties of the orthogonal polynomials used. We also present identities involving the recursion coefficients, necessary to solve the triple-scaling limit.

- In sect. 2, we give the planar limit of the string equations. We discuss the identification of the critical models with singularities in two-dimensional mappings. We also give a brief discussion of the saddle point analysis, which provides a complementary description of the planar limit.

- Triple-scaling ansätze are introduced in sect. 3. We illustrate the construction of scaling solutions with an explicit example. For this example, the associated two-dimensional mapping can be decomposed into two separate one-dimensional singularities, and the resulting scaling solution involves two decoupled ordinary differential equations in two independent variables.

- In sect. 4, we provide examples of new scaling equations, whose general form is a single ordinary differential equation which includes the second scaling variable as a parameter. The scaling functions satisfy finite difference equations for shifts in this second variable. Two of these examples are related to the simplest intrinsically two-dimensional singularity, the Whitney pleat[11].

- Sect. 5 displays a new family of multicritical models for which i) the associated singularities are again intrinsically two-dimensional, ii) the scaling equations are two coupled partial differential equations in the two scaling variables, and iii) the equations admit two qualitatively different weak-coupling expansions.

- Some concluding remarks and conjectures are given in sect. 6.

\section{Notation and polynomial recursion relations}

We define first matrices of the following form:

$$
H \equiv\left(\begin{array}{cc}
0 & T \\
T^{\dagger} & 0
\end{array}\right),
$$

where $T$ is an $N \times M$ matrix with complex entries. In the following, we will assume that $N \geq M$ without any loss of generality. These matrices have a natural action of $\mathrm{U}(N) \times \mathrm{U}(M)$, which can be used to bring $T$ to the form

$$
T=\left(\begin{array}{c}
X_{M} \\
0_{(N-M) \times M}
\end{array}\right),
$$

where $X_{M}$ is a real diagonal $M \times M$ matrix $\operatorname{diag}\left(x_{1}, x_{2}, \ldots, x_{M}\right)$. Analogous statements can be made about matrices with real or quaternionic entries, as well as circular ensembles obtained by exponentiating such matrices[2]. All the steps in the following can be repeated for these ensembles as well, albeit with some technical complications[12,13,14]. 
We wish to study the free energy of the partition function defined as

$$
\mathcal{Z} \equiv \int \mathrm{d} H \exp \left(-\beta \operatorname{tr} V\left(H^{2}\right)\right)
$$

where $\mathrm{d} H$ is the Haar measure, and the matrix potential is a polynomial of the form: $V\left(H^{2}\right)=\sum_{p=1}^{L} \frac{a_{p}}{2 p} H^{2 p}$. (Note that tr $H^{2 k+1}=0$.) Such matrix integrals lead to theories of random surfaces, via Feynman diagram expansions[15]. The invariance of $\mathcal{Z}$ under $\mathrm{U}(N) \times \mathrm{U}(M)$ leads to the following integral:

$$
\mathcal{Z}=\int_{-\infty}^{\infty} \prod_{i=1}^{M}\left[\mathrm{~d} x_{i} \exp \left(-2 \beta V\left(x_{i}^{2}\right)\right)\right] \prod_{i=1}^{M}\left|x_{i}\right|^{2(N-M)+1} \prod_{1 \leq i<j \leq M}\left(x_{i}^{2}-x_{j}^{2}\right)^{2}
$$

where overall factors, which do not affect the critical behavior, have been dropped. From the Jacobian appearing in (3), it is clear that the partition function is a function of $P \equiv N-M$, and $M$, independently.

An obvious change of variables from $x$ to $y=x^{2}$ simplifies the analysis of (3). It is then clear that we need orthogonal polynomials on a half-line, and relations between polynomials for measures related by the insertion of powers of $y$. Some of these are derived in Chihara[16]. Let

$$
\mathrm{d} \mu[l] \equiv y^{l} \exp (-2 \beta V(y))
$$

where one assumes the measure has support in $[0, \infty){ }^{\dagger}$ Denote orthogonal polynomials for such a measure by $P_{n}^{(l)}(y)$, such that

$$
\int_{0}^{\infty} \mathrm{d} \mu[l] P_{n}^{(l)}(y) P_{m}^{(l)}(y)=\delta_{n, m} h_{m}^{(l)}
$$

where $P_{n}^{(l)}(y) \equiv y^{n}+$ lower order terms. Multiplying by $y$ yields the usual three-term recursion relation

$$
y P_{n}^{(l)}(y)=P_{n+1}^{(l)}(y)+S_{n}^{(l)} P_{n}^{(l)}(y)+\alpha_{n}^{(l)} P_{n-1}^{(l)}(y)
$$

where $\alpha_{n}^{(l)}=h_{n}^{(l)} / h_{n-1}^{(l)}$. There are also simple recursion relations relating polynomials defined for measures with different values of $l$

$$
\begin{aligned}
P_{n}^{(l)}(y) & =P_{n}^{(l+1)}(y)+\phi_{n}^{(l)} P_{n-1}^{(l+1)}(y), \\
y P_{n}^{(l+1)}(y) & =P_{n+1}^{(l)}(y)+\theta_{n+1}^{(l)} P_{n}^{(l)}(y),
\end{aligned}
$$

where

$$
\phi_{n}^{(l)}=\frac{h_{n}^{(l)}}{h_{n-1}^{(l+1)}} \quad \text { and } \quad \theta_{n+1}^{(l)}=\frac{h_{n}^{(l+1)}}{h_{n}^{(l)}}=-\frac{P_{n+1}^{(l)}(0)}{P_{n}^{(l)}(0)}
$$

$\dagger$ The measure will be divergent at the latter end-point for several critical models, in which case the following identities must be regarded as analytically continued from the convergent cases. 
Our scaling analysis in the following will be made in terms of the recursion coefficients, $\phi_{n}^{(l)}$ and $\theta_{n}^{(l)}$. The coefficients appearing in (4) are easily determined by

$$
S_{n}^{(l)}=\theta_{n+1}^{(l)}+\phi_{n}^{(l)} \quad \text { and } \quad \alpha_{n}^{(l)}=\theta_{n}^{(l)} \phi_{n}^{(l)} .
$$

One can show that $\theta_{n}^{(l)}$ and $\phi_{n}^{(l)}$ appear as recursion coefficients in the formula analogous to (4) for orthogonal polynomials defined for $x$, rather than $y$.

Further useful relations between the coefficients are

$$
\begin{aligned}
& \phi_{n}^{(l)}-\phi_{n-1}^{(l+1)}=\theta_{n}^{(l+1)}-\theta_{n}^{(l)}, \\
& \phi_{n}^{(l-1)} \theta_{n+1}^{(l-1)}=\phi_{n}^{(l)} \theta_{n}^{(l)} .
\end{aligned}
$$

These relations are independent of the matrix potential, $V(y)$, and their derivation is elementary, so we shall not give it here. One can think of $(8,9)$ are discrete analogues of 'zero-curvature' conditions (i.e., they ensure that the operations of shifting the indices $l$ and $n$ commute). Their importance in the solution of these models is quite considerable. Indeed in section 5, we shall find a KdV-like partial differential originating from the study of these relations. To this equation are associated an infinite set of triple-scaling multicritical points. These identities become trivial in the planar limit, with $l=P, n=M$, and $\beta \rightarrow \infty$ keeping $P / \beta$ and $M / \beta$ finite (see sect. 3).

Using the properties listed above, one can derive two independent 'string equations':

$$
\begin{aligned}
\frac{2 n+l+1}{2 \beta} h_{n}^{(l)} & =\int \mathrm{d} \mu[l] y V^{\prime}(y) P_{n}^{(l)}(y) P_{n}^{(l)}(y) \\
\frac{n}{2 \beta} h_{n-1}^{(l+1)} & =\int \mathrm{d} \mu[l] V^{\prime}(y)\left(P_{n}^{(l)}(y)^{2}+\theta_{n}^{(l)} P_{n}^{(l)}(y) P_{n-1}^{(l)}(y)\right) .
\end{aligned}
$$

The independence of these equations is obvious since $l$ and $n$ are completely independent parameters. Using the operator techniques introduced in Ref. 17, one can reexpress these identities as

$$
\begin{aligned}
& \frac{2 n+l+1}{2 \beta}=\int_{0}^{2 \pi} \frac{\mathrm{d} \lambda}{2 \pi} \bar{y}\left(n-i \partial_{\lambda}\right) V^{\prime}\left[\bar{y}\left(n-i \partial_{\lambda}\right)\right] \cdot 1_{l} \\
& \frac{n}{2 \beta}=\phi_{n}^{(l)}\left(\int_{0}^{2 \pi} \frac{\mathrm{d} \lambda}{2 \pi} V^{\prime}\left[\bar{y}\left(n-i \partial_{\lambda}\right)\right] \cdot 1_{l}+\theta_{n}^{(l)} \int_{0}^{2 \pi} \frac{\mathrm{d} \lambda}{2 \pi} e^{-i \lambda} V^{\prime}\left[\bar{y}\left(n-1-i \partial_{\lambda}\right)\right] \cdot 1_{l}\right)
\end{aligned}
$$

where

$$
\bar{y}\left(n-i \partial_{\lambda}\right)=e^{i \lambda}+\hat{\theta}\left(1+n-i \partial_{\lambda}\right)+\hat{\phi}\left(n-i \partial_{\lambda}\right)+e^{-i \lambda} \hat{\theta}\left(n-i \partial_{\lambda}\right) \hat{\phi}\left(n-i \partial_{\lambda}\right)
$$

The subscript $l$ on $1_{l}$ indicates that all of the recursion coefficients carry the same superscript (l) (i.e., $\left.\hat{\theta}\left(n-i \partial_{\lambda}\right) \cdot 1_{l}=\theta_{n}^{(l)}\right)$. Note then that these identities only involve coefficients with differing subscripts. These equations are also non-trivial in the planar limit. Both of these features contrast them from the potential independent identities, (8) and (9). 


\section{Planar limit}

We begin by considering the planar limit, in which only the contributions of surfaces with spherical topology survive. Explicitly in the recursion coefficient identities above, we set $l=P$ and $n=M$, and consider the limit $\beta \rightarrow \infty$ keeping $g \equiv N / \beta$ and $q \equiv M / \beta$ finite. We assume that the recursion coefficients have a smooth limit, $\theta_{M \pm j}^{(P \pm i)} \rightarrow \theta$ and $\phi_{M \pm j}^{(P \pm i)} \rightarrow \phi$. Clearly in this case, eq.'s $(8,9)$ vanish trivially. On the other hand, eq.'s $(12,13)$ become algebraic, and can be interpreted as giving the extrema of the following functional

$$
W(\theta, \phi)=U(\theta, \phi)-g \ln \theta-q \ln \phi
$$

where

$$
U(\theta, \phi)=2 \int_{0}^{2 \pi} \frac{\mathrm{d} \lambda}{2 \pi} V\left(e^{i \lambda}+\theta+\phi+e^{-i \lambda} \theta \phi\right)
$$

Explicitly, given a potential $V(y)=\sum_{p=1}^{L} a_{p} y^{p} /(2 p)$, one finds

$$
U(\theta, \phi)=\sum_{p=1}^{L} \frac{a_{p}}{p} \sum_{k=0}^{p}\left(\begin{array}{l}
p \\
k
\end{array}\right)^{2} \theta^{k} \phi^{p-k}
$$

Recalling the relations (7) of $\theta$ and $\phi$ to the usual recursion coefficients in (4), note the similarity of $U$ to the functional $\Omega$ introduced in Ref. 17 .

The critical points can be identified as follows: The planar equations take the form

$$
g=\theta \partial_{\theta} U(\theta, \phi) \quad \text { and } \quad q=\phi \partial_{\phi} U(\theta, \phi)
$$

In the case of hermitian matrix models[17], one finds a single equation $g=g(\alpha)$. In that case, the critical values of the coupling constants are chosen such that $\frac{\mathrm{d}^{i} g}{\mathrm{~d} \alpha^{i}}=0$ for $i=0, \ldots, k$. Thus the critical points are identified as points where $\alpha=\alpha(g)$ is nonanalytic, or alternatively as points where the map $g \mapsto \alpha$ is singular. Given this point of view, it is natural to identify the the critical points in the present case as points where the two-dimensional map $(g, q) \mapsto(\theta, \phi)$ becomes singular. Such points occur at the vanishing of the Jacobian determinant[11]:

$$
0=|J|=\left|\begin{array}{ll}
\frac{\partial g}{\partial \theta} & \frac{\partial g}{\partial \phi} \\
\frac{\partial q}{\partial \theta} & \frac{\partial q}{\partial \phi}
\end{array}\right|
$$

Multicritical behavior is produced by demanding the vanishing of various higher derivatives of $g$ and $q$. In terms of the functional $W$, these (multi)critical points correspond to degenerate extrema. In the scaling limit which will be of interest below, one does not focus on the degenerate point exactly. Rather the singularity is resolved by also perturbing $g$ and $q$ near their critical values[1]. Schematically one might find

$$
W \approx \sum A_{i}\left(\hat{\phi}_{c}-\hat{\phi}\right)^{p_{i}}\left(\hat{\theta}_{c}-\hat{\theta}\right)^{k_{i}}-\left(\hat{g}_{c}-\hat{g}\right)\left(\hat{\theta}_{c}-\hat{\theta}\right)-\left(\hat{q}_{c}-\hat{q}\right)\left(\hat{\phi}_{c}-\hat{\phi}\right)
$$


where $p_{i}$ and $k_{i}$ are integers, and $A_{i}$, arbitrary constants. The variables in (17) are denoted with ^ to indicate that they can be arbitrary linear combinations of $\phi, \theta, g$ and $q$. Generically the singularity will most naturally be parameterized in terms of various linear combinations, rather than $\phi, \theta, g$ and $q$ directly. This analysis of the critical points is identical to that for coupled vector models[7]. In that case, the functional $W$ is replaced by an effective potential $\widehat{V}$, which is of direct physical relevance in those models. The coefficients of the polynomial terms in $\widehat{V}$ are all completely independent. In contrast, (14) shows that many of these coefficients in $W$ are directly related. This restricts the possible singularities that arise. The essential two-dimensional character of the critical points leads to a much richer structure than appears for ensembles of square matrices, though. We do not have a complete classification of all possible singularities, but in the following, we will provide various explicit examples which illustrate very distinct scaling behaviors. Each of these examples extend to an infinite family of higher multicritical models, some of which will be discussed further in Ref. 18.

Before proceeding with examples of triple-scaling behavior, we would like to briefly discuss the saddle point analysis, because it provides a more physically intuitive description of the planar limit, and of the critical behavior. First, we mention that using the saddle point approximation, the planar limit of rectangular matrix models with non-vanishing $a_{1}$ and $a_{2}$ was completely solved in Ref. [8]. The following discussion applies for general potentials.

The partition function can be written as

$$
\mathcal{Z}_{N, M}=\int_{0}^{\infty} \prod_{i=1}^{M}\left(\mathrm{~d} y_{i} y_{i}^{N-M} \exp \left[-2 \beta V\left(y_{i}\right)\right]\right) \prod_{1 \leq i<j \leq M}\left(y_{i}-y_{j}\right)^{2}
$$

with $V(y)=\sum_{p=1}^{L} a_{p} y^{p} /(2 p)$. The entire integrand can be expressed as $\exp \left(-\beta^{2} E\right)$, where

$$
\beta E\left(\left\{y_{i}\right\}\right)=2 \sum_{i=1}^{M} V\left(y_{i}\right)-\frac{N-M}{\beta} \sum_{i=1}^{M} \ln y_{i}-\frac{1}{\beta} \sum_{i \neq j ; i, j=1}^{M} \ln \left|y_{i}-y_{j}\right| .
$$

The last term above arises from the Vandermonde factor in the Jacobian, and can be interpreted as a repulsive electrostatic potential between different particles at $y_{i}$ in a onedimensional system. The second term also coming from the Jacobian, represents a further electrostatic repulsion away from the origin, whose strength is controlled by $P=N-M$. This repulsion combines with the external potential $V$ to balance the Coulomb repulsion of the particles, and confine them to some interval $[a, b]$ at equilibrium. We will assume $0 \leq a \leq b .^{\dagger}$ Evaluating (18) for large $\beta$ in the saddle point approximation, requires finding a configuration of 'eigenvalues' satisfying

$$
\frac{\partial V\left(y_{i}\right)}{\partial y_{i}}-\frac{N-M}{2 \beta y_{i}}-\frac{1}{\beta} \sum_{j \neq i ; j=1}^{M} \frac{1}{y_{i}-y_{j}}=0 .
$$

$\dagger$ The following analysis is only correct for $a=0$ if the eigenvalue density vanishes there. A proper treatment for a nonvanishing density at the origin is most easily done in terms of the original variables $x$ rather than $y[19,3]$. 
Defining a density of eigenvalues, $\rho(y)$, in the usual manner[20], one finds

$$
V^{\prime}(y)-\frac{g-q}{2 y}=q f_{a}^{b} \frac{1}{y-z} \rho(z) \mathrm{d} z .
$$

Solving this equation for $\rho(y)$ is an example of the Hilbert problem on an arc[21]. The results can be expressed as constraints on the function

$$
F(z)=\frac{1}{q}\left(V^{\prime}(z)-\frac{g-q}{2 z}-u(z) \sqrt{(z-b)(z-a)}\right)
$$

where $u(z)=\sum_{k=-1}^{L-2} h_{k} z^{k}$. The constants $h_{k}, a$ and $b$ are fixed by demanding that $F(z) \rightarrow$ $1 / z$ as $|z| \rightarrow \infty$, and that $F(z)$ remains finite as $|z| \rightarrow 0$. The eigenvalue density on $[a, b]$ is then given by

$$
\rho(y)=\frac{1}{\pi q} u(y) \sqrt{(b-y)(y-a)} .
$$

By examining the equations fixing $a$ and $b$, one finds that they are identical to the planar string equations (15) when one makes the identification

$$
\theta=\frac{1}{4}(\sqrt{b}+\sqrt{a})^{2} \quad \text { and } \quad \phi=\frac{1}{4}(\sqrt{b}-\sqrt{a})^{2} .
$$

Further using this identification, the coefficients $h_{k}$ can be written as

$$
h_{k}=\frac{1}{2} \sum_{p=0}^{L-2-k} a_{p+k+2} \sum_{l=0}^{p}\left(\begin{array}{l}
p \\
l
\end{array}\right)^{2} \theta^{l} \phi^{p-l}
$$

which is a form similar to that for $U(\theta, \phi)$ in (14).

The aim is to evaluate the planar free energy,

$$
E_{0}=q \int_{a}^{b} \mathrm{~d} y \rho(y)[2 V(y)-(g-q) \ln |y|]-q^{2} \int_{a}^{b} \mathrm{~d} z \mathrm{~d} y \rho(z) \rho(y) \ln |z-y|
$$

Integrating (20), one observes that

$$
q \int_{a}^{b} \mathrm{~d} z \rho(z) \ln |y-z|=V(y)-\frac{g-q}{2} \ln |y|-K
$$

where one can set

$$
K=V(a)-\frac{g-q}{2} \ln |a|-q \int_{a}^{b} \mathrm{~d} z \rho(z) \ln |z-a|
$$

Then

$$
E_{0}=q \int_{a}^{b} \mathrm{~d} y \rho(y)\left[V(y)-\frac{g-q}{2} \ln |y|-q \ln |y-a|\right]+q V(a)-\frac{g-q}{2} q \ln |a| .
$$


Critical behaviour corresponds to a nonanalytic dependence of the free energy $E_{0}$ on the coupling constants in $V$ or $q$. For fixed couplings, this can be expressed as nonanalyticity in $g$ and $q$. Roughly we want

$$
E_{\text {sing }} \sim\left(\hat{g}_{c}-\hat{g}\right)^{\gamma}\left(\hat{q}_{c}-\hat{q}\right)^{\delta} .
$$

Such nonanalytic behavior arises at the melting phase transition, where the eigenvalue density (21) acquires zeroes at the boundaries, $a$ and/or $b$, beyond those evident in (21). Using eq.'s $(14,15,22,23)$, this mechanistic description is related to (16) by observing that $|J|=4 a b u(a) u(b)$.

\section{Triple-scaling and Split Singularities}

In this section, we discuss triple-scaling for the case of split singularities. We use the latter term to describe two-dimensional singularities which can be separated into two independent one-dimensional singularities. In double-scaling physics[1], many relations between quantities associated with critical behaviour follow from the basic ansatz that

$$
\frac{N}{\beta}=\left[\frac{N}{\beta}\right]_{c}-\beta^{\nu-1} t
$$

where $t$ is the double-scaling variable associated with the continuum theory. So long as $\nu>0$, it is reasonable to assume that the difference equations that characterize the matrix model at finite $N$ are transmuted into differential equations in the variable $t$ as $\beta \rightarrow \infty$. In triple-scaling physics, as in Ref. 7 , we have two variables in the planar limit that can play the rôle of $N$, and thus (25) is naturally extended to

$$
\frac{X}{\beta}=\left[\frac{X}{\beta}\right]_{c}-\beta^{\nu-1} t \quad \text { and } \quad \frac{Y}{\beta}=\left[\frac{Y}{\beta}\right]_{c}-\beta^{\hat{\nu}-1} s
$$

where $X$ and $Y$ are linearly independent combinations of $M$ and $N$ determined for a given singularity.

In our first example, rather than imposing simply $|J|=0$ as in (16), we require the stronger condition that the individual derivatives in the Jacobian matrix all vanish (i.e., $\left.\frac{\partial g}{\partial \theta}=0=\frac{\partial g}{\partial \phi}=\frac{\partial q}{\partial \theta}=\frac{\partial q}{\partial \phi}\right) .^{\dagger}$ Thus the expansion of the planar equations (15) about the critical point will begin at second order in both variations: $\Delta \phi=\phi-\phi_{c}$ and $\Delta \theta=\theta-\theta_{c}$. One finds that when any two of the derivatives are set to vanish, the remaining two vanish automatically. Tuning for this critical point, therefore, imposes fewer constraints on the matrix potential than one might expect. The minimal potential only requires that the first three coefficients be nonvanishing.

There is enough freedom in the planar equations to rescale the variables at any critical point to set $g_{c}=[N / \beta]_{c}=1$ and $\theta_{c}=1$. We make this choice. We also set $\phi_{c}=y^{2}$ since

$\dagger$ Critical models with the simplest tuning to produce $|J|=0$ have more involved scaling solutions - see the following sections. 
it is non-negative - see either (22) or (6). With these choices, the matrix potential can be tuned so that the desired derivatives vanish by setting:

$$
a_{1}=-3 \frac{1+y^{4}}{3 y^{2}-1} ; \quad a_{2}=3 \frac{1+y^{2}}{3 y^{2}-1} ; \quad a_{3}=-\frac{1}{3 y^{2}-1} .
$$

One finds that $q_{c}=[M / \beta]_{c}=y^{4}\left(3-y^{2}\right) /\left(3 y^{2}-1\right)$. $\phi_{c}$ remains free-different values correspond to approaching the large- $N$ limit with rectangular matrices of different proportions. $g_{c}=1 \geq q_{c} \geq 0$ implies that $3 \geq y^{2} \geq 1$. Thus the critical potential is unbounded from below. Expanding (15) about the critical point, to leading order

$$
\begin{aligned}
& \Delta g=-\frac{3}{3 y^{2}-1}\left(\Delta \phi^{2}+2 \Delta \phi \Delta \theta+y^{2} \Delta \theta^{2}\right) \\
& \Delta q=-\frac{3}{3 y^{2}-1}\left(\Delta \phi^{2}+2 y^{2} \Delta \phi \Delta \theta+y^{2} \Delta \theta^{2}\right)
\end{aligned}
$$

where $\Delta g=g-g_{c}, \Delta q=q-q_{c}$. Eq. (27) can be rewritten as

$$
y \Delta g \pm \Delta q=-\frac{3}{3 y^{2}-1}(y \pm 1)(y \Delta \theta \pm \Delta \phi)^{2},
$$

so one has two independent quadratic singularities.

Eq. (28) also shows that the natural parameters in which to construct the triple scaling solution are $y g \pm q$ and $y \theta \pm \phi$. First for (26), we define $t, s$ by

$$
\begin{aligned}
& \frac{y N+M}{\beta}=\left[\frac{y N+M}{\beta}\right]_{c}-\beta^{\nu-1} t \\
& \frac{y N-M}{\beta}=\left[\frac{y N-M}{\beta}\right]_{c}-\beta^{\nu-1} s .
\end{aligned}
$$

The same exponents are chosen in both expressions, since the independent singularities are the same. With these ansätze, one can Taylor expand the differences in $M$ and $P$ that arise in eq.'s $(8,9,12,13)$ using

$$
\partial_{M}=-(y+1) \delta \partial_{t}-(y-1) \delta \partial_{s} \quad \text { and } \quad \partial_{P}=-y \delta \partial_{t}-y \delta \partial_{s}
$$

where $\delta=\beta^{-\nu}$. The natural scaling ansätze for the recursion coefficients are then

$$
\begin{aligned}
& y \theta_{M+n}^{(P+l)}+\phi_{M+n}^{(P+l)}=y(1+y)-\exp \left[-\Delta_{l, n}\right] \sum_{q=2} \delta^{q} h_{q}(t, s) \\
& y \theta_{M+n}^{(P+l)}-\phi_{M+n}^{(P+l)}=y(1-y)-\exp \left[-\Delta_{l, n}\right] \sum_{q=2} \delta^{q} k_{q}(t, s) .
\end{aligned}
$$

where $\Delta_{l, n} \equiv \delta\left[n\left((y+1) \partial_{t}+(y-1) \partial_{s}\right)+l y\left(\partial_{t}+\partial_{s}\right)\right]$.

We find that $\nu=\frac{1}{5}$. The potential independent equations $(8,9)$ yield:

$$
\partial_{s} h_{2}(t, s)=0=\partial_{t} k_{2}(t, s) .
$$


Then $(12,13)$ give

$$
\begin{aligned}
& s=3 \frac{y-1}{3 y^{2}-1} k_{2}(s)^{2}-\frac{y^{2}(y-1)^{2}}{3 y^{2}-1} \partial_{s}^{2} k_{2}(s), \\
& t=3 \frac{y+1}{3 y^{2}-1} h_{2}(t)^{2}-\frac{y^{2}(y+1)^{2}}{3 y^{2}-1} \partial_{t}^{2} h_{2}(t) .
\end{aligned}
$$

This result, that the scaling solution is governed by two independent Painleve I equations, may have been anticipated given that the critical point consists of two separate quadratic singularities. Such one-dimensional singularities lead to the Painleve I equation for hermitian matrix models[1]. A priori though, there is no reason that the derivative terms in the differential scaling equations should respect this splitting of the singularity. The crucial result implying this separation is (32), which is solved by $k_{2}=k_{2}(s)$ and $h_{2}=h_{2}(t)$.

What is the free energy of the matrix model? $\mathcal{Z}_{M+1, P} / \mathcal{Z}_{M, P}=(M+1) h_{M}^{(P)}$ and (6) imply that

$$
\begin{gathered}
\alpha_{M}^{(P)} \approx \frac{\mathcal{Z}_{M+1, P} \mathcal{Z}_{M-1, P}}{\mathcal{Z}_{M, P}^{2}} \approx \exp \left(-\partial_{M}^{2} F\right) \\
\theta_{M+1}^{(P)} \approx \frac{\mathcal{Z}_{M+1, P+1} \mathcal{Z}_{M, P}}{\mathcal{Z}_{M, P+1} \mathcal{Z}_{M+1, P}} \approx \exp \left(-\partial_{M} \partial_{P} F\right) .
\end{gathered}
$$

Combining these results with (30) and (31), one is able to show: First, $\partial_{t} \partial_{s} F$ is a constant. Then up to some possible analytic terms, one finds

$$
\partial_{s}^{2} F=\frac{k_{2}(s)}{2 y^{2}(y-1)} \quad \text { and } \quad \partial_{t}^{2} F=\frac{h_{2}(t)}{2 y^{2}(y+1)} .
$$

Thus the nonanalytic contributions to the free energy are divided into two completely separate pieces, both with asymptotic expansions identical to that found for ordinary twodimensional gravity[1]. The relevant expansion parameters in the present case are $t^{-\frac{5}{2}}, s^{-\frac{5}{2}}=$ $\beta^{2}\left(\frac{y N \pm M}{\beta}-\left[\frac{y N \pm M}{\beta}\right]_{c}\right)^{-\frac{5}{2}}$. The appearance of two scaling functions should not be confused with the doubling found in hermitian matrix models[22], since in the present case they are functions of completely independent parameters. Furthermore, both scaling equations are inhomogenous in the scaling variables without the appearance of any nonuniversal coefficients[23]. By rescaling $h_{2}, k_{2}, t$ and $s$ appropriately, the results for all different values of $\phi_{c}$ can be reduced to

$$
\begin{array}{rlrl}
s & =k_{2}(s)^{2}-\frac{1}{6} \partial_{s}^{2} k_{2}(s) ; & & \partial_{s}^{2} F=k_{2}(s) \\
t & =h_{2}(t)^{2}-\frac{1}{6} \partial_{t}^{2} h_{2}(t) ; & \partial_{t}^{2} F=h_{2}(t) .
\end{array}
$$

Therefore all of these models with matrices of different proportions produce identical continuum physics.

The natural generalization for these split singularities would be to tune the potential to produce separate one-dimensional singularities of independent orders. However, such 
singularities do not arise in the triple-scaling solution of rectangular matrix models. The example given here does generalize to a family of split singularities of the same order,

$$
y \Delta g \pm \Delta q \propto(y \Delta \theta \pm \Delta \phi)^{n}
$$

where $n$ is a positive integer. Implicitly we include the 'topological' model with $n=1$, but of course this does not correspond to a singular mapping. The only assumption involved in producing (32) was that the scaling parameters appear with the same exponents. Since those results were derived from the potential independent equations $(8,9)$, they will also apply for the higher singularities considered in (34). Hence one will recover $k_{2}=k_{2}(s)$ and $h_{2}=h_{2}(t)$ for all of these critical points, and the scaling behaviour will then be governed by two independent ordinary differential equations of the form appearing in double-scaled matrix models. Note that at least up to $n=4$, we found that tuning to increase the power of the singularity in (34) by one, only requires introducing two extra coefficients in the minimal potential. This fact is an example of how the interrelatedness of the coefficients in the functional $U(\theta, \phi)$ in (14) restricts the set of singularities that can be attained in these matrix models.

At the beginning, we found that $g_{c}>q_{c}>0$ required $3>y^{2}>1$. One may ask what happens at the end points of this interval. With $y^{2}=3$, the previous analysis and solution remain unchanged. It is only an exceptional point since $q_{c}=[M / \beta]_{c}=0$. This does not mean that the number of eigenvalues is zero or even finite. From (26), one finds that $M=\beta^{\frac{1}{5}}(s-t) / 2$. Therefore the number of eigenvalues diverges, but at a much slower rate than either $N$ or $\beta$. At $y^{2}=1$, the previous analysis fails. One sees in (28) that one of $y \Delta g \pm \Delta q$ vanishes to the given order in the expansion of planar equations. Hence the nature of the singularity changes at this point, and higher order terms must be included in the expansion (27) for a proper analysis. In this case, the scaling solution is similar to that presented in the section 5, as will be discussed in Appendix A.

Consider the saddle point solution for the critical point solved here. Using (21) and (23), the eigenvalue density has two extra zeroes, one at each of the two boundaries (i.e., $u(a)=0=u(b))$. The saddle point analysis leading to this result is not valid in the present case as is evident from (22), which requires $\theta_{c} \geq \phi_{c}$. As discussed above, the scaling analysis is valid for $\phi_{c}=y^{2}>1=\theta_{c}$. Therefore the triple scaling solution presented here corresponds to a more involved analytic continuation of the matrix model than considered for the saddle point analysis presented in the previous section. This complication does not hold for all of the split singularity critical points. One finds that $\theta_{c} \geq \phi_{c}$ for the odd powers in (34).

\section{Parametric Solutions}

In this section we exhibit some novel critical phenomena, associated with intrinsically two-dimensional singularities. We begin with a general analysis of the planar limit to identify critical points for the simplest nontrivial potential, $V(y)=a_{1} y / 2+a_{2} y^{2} / 4$. We focus on the scaling solution of two higher order multicritical points. The scaling analysis for the generic 
points will be presented in the following section. These multicritical points correspond to deviations from the two extreme limits of vector models and square matrix models, and only one of the large- $N$ parameters, $M$ or $P$, diverges while the other remains finite. The recursion equations, presented in section 1 , lead to a coupled set of differential and finite difference equations. The difference equations relate scaling functions at different values of the finite parameter. The finite parameter also appears as an extra constant in the differential scaling equations. Therefore we refer to the results for these critical points as parametric solutions. We shall show that the new methods introduced in this paper lead to results that both include, and extend, known results[24,2,3,4,5].

For the simplest nontrivial potential, the vanishing of the Jacobian determinant (16) becomes

$$
|J|=a_{1}^{2}+4 a_{1} a_{2}\left(\phi_{c}+\theta_{c}\right)+4 a_{2}^{2}\left(\phi_{c}^{2}+\phi_{c} \theta_{c}+\theta_{c}^{2}\right)=0 .
$$

We normalize $g_{c}=1=\theta_{c}$ and set $\phi_{c}=y^{2}$. In this case, (35) yields

$$
a_{1}=2 \frac{1+y+y^{2}}{2 y+1} ; \quad a_{2}=-\frac{1}{2 y+1} .
$$

(A second solution, which yields identical results, is produced by replacing $y \rightarrow-y$.) Note that none of the individual derivatives appearing in the Jacobian matrix vanish at this critical point. The numerator in $a_{1}$ is a positive definite function of $y$, and it is straightforward to show $a_{1} / a_{2} \leq-3 / 2$. This implies, in particular, that $\operatorname{sgn} a_{1}=-\operatorname{sgn} a_{2}$ for critical behaviour. One finds that $q_{c}=y^{3}(2+y) /(2 y+1)$. Requiring that $g_{c}=1 \geq q_{c} \geq 0$, one finds two possible ranges for $y$ : $0 \leq y \leq 1$ and $-2 \leq y \leq-1$. Only the former range satisfies $\theta_{c} \geq \phi_{c}$, while only the latter corresponds to a stable potential. We define $\Delta \theta, \Delta \phi, \Delta g, \Delta q$ as in the previous section, to obtain

$$
\begin{aligned}
& \Delta g=-\frac{2}{2 y+1}(\Delta \phi-y \Delta \theta)-\frac{1}{2 y+1}\left(\Delta \theta^{2}+2 \Delta \theta \Delta \phi\right) \\
& \Delta q=\frac{2 y}{2 y+1}(\Delta \phi-y \Delta \theta)-\frac{1}{2 y+1}\left(\Delta \phi^{2}+2 \Delta \theta \Delta \phi\right) .
\end{aligned}
$$

This singularity has two notable features: The linear combination $\hat{q}=q+y g$ is distinguished since the expansion begins at quadratic order for this parameter. Therefore one has $\frac{\partial \hat{q}}{\partial \theta}=$ $0=\frac{\partial \hat{q}}{\partial \phi}$. Eq. (36) also singles out $\hat{\phi}=\phi-y \theta$ as a special linear combination of the recursion coefficients. This is because the variations of any linear combination of $g$ and $q$, except for $\hat{q}$, about the critical point are linear in $\Delta \hat{\phi}$. The universal behavior is independent of the choice of the remaining variables, so it is best made on the basis of simplifying the calculations. At present, we wish to focus on exceptional points where the nature of the singularity changes. In the previous section, we saw that such changes may occur at the end points in the allowed range of $y$. In the present example that there are two cases of interest: i) $y=0$ and ii) $y=-1$. This fact will be verified in the analysis of the generic points in sect. 5 .

(i) $y=0: \quad g_{c}=1, q_{c}=0, \theta_{c}=1, \phi_{c}=0, a_{1}=2, a_{2}=-1$ 
The fact that $\phi_{c}=0$ has some important implications. First from (22), the width of the eigenvalue distribution in the saddle point analysis has shrunk to zero. Thus the analysis given there is inappropriate for this particular critical point, but it suggests that the correct saddle point analysis is that given in [3] for vector models. In the saddle point analysis for those models, $M / \beta \rightarrow 0$ so that the eigenvalues accumulate at a single point since their electrostatic repulsion is unable to keep them apart. One can verify that the critical values given above correspond to one of the critical points for the vector models. This would imply that $M$ should remain finite in the present case. This conclusion is further supported by the fact that $\alpha_{c}=\theta_{c} \phi_{c}=0$. Given the usual connection in (33) of $\alpha$ to the free energy for a standard scaling solution, $\alpha_{c}=0$ suggests that differences in $M$ do not give rise to derivatives suppressed by powers of $\beta$ in the scaling limit.

Returning to the expansion about the critical point, one has $\hat{q}=q$ and $\hat{\phi}=\phi$. It is convenient to choose $\theta$ and $P / \beta \equiv(N-M) / \beta=g-q$ as the remaining variables with which to describe the singularity. Now (36) reduces to

$$
\Delta(g-q)=-2 \Delta \phi-\Delta \theta^{2}, \quad \Delta q=-2 \Delta \theta \Delta \phi
$$

where a term of order $\Delta \phi^{2}$ has been dropped from both expressions, since in the scaling limit they are higher order. Rewriting (37) as $\Delta q=\Delta \theta^{3}+\Delta \theta \Delta(g-q)$, one can recognize this singularity as a Whitney pleat[11].

Let us introduce scaling variables as in $(29,30,31)$,

$$
\begin{aligned}
\frac{P}{\beta} & \equiv 1-\beta^{\nu-1} t, \\
\frac{M}{\beta} & \equiv \beta^{\hat{\nu}-1} s,
\end{aligned}
$$

and

$$
\begin{aligned}
\theta_{m}^{(P+l)} & \equiv 1-\exp \left[-l \beta^{-\nu} \partial_{t}\right] \beta^{-\mu} f_{m}(t), \\
\phi_{m}^{(P+l)} & \equiv-\exp \left[-l \beta^{-\nu} \partial_{t}\right] \beta^{-\hat{\mu}} g_{m}(t) .
\end{aligned}
$$

Following the previous discussion, we have not introduced $m=M+n$ or derivatives with respect to $s$ for differences in $m$. From (37), we know the exponents are related as $\hat{\mu}=2 \mu$, $\nu=1-2 \mu$ and $\hat{\nu}=1-3 \mu$, so it remains to find $\mu$. For this, we turn to $(8,9)$, and find

$$
\begin{aligned}
& 0=g_{m}-g_{m-1}+\partial_{t} f_{m}, \\
& 0=g_{m-1}\left(f_{m-1}-f_{m}\right)+\partial_{t} g_{m-1},
\end{aligned}
$$

provided that $\mu=\nu$, which in turn implies $\mu=\frac{1}{3}$, and $\hat{\nu}=0$. With the vanishing of this last exponent, one sees that as expected $M / \beta$ does not scale, but rather (38) would give $M=s$. Thus it is inappropriate to replace discrete differences in the subscripts in (39) with derivatives, since the derivatives would not be suppressed by negative powers of $\beta$, and we would have to keep track of arbitrarily high powers of $\partial_{s}$. 
Applying our ansätze to $(12,13)$ to complete the scaling analysis, we find

$$
\begin{aligned}
& 0=m+g_{m}\left(f_{m}+f_{m+1}\right) \\
& 0=t+g_{m}+g_{m-1}-f_{m}^{2} .
\end{aligned}
$$

Combining these results with (40), we derive a single equation in terms of $f_{m}$ only, which takes the surprising form

$$
\partial_{t}^{2} f_{m}-2 f_{m}^{3}+2 t f_{m}=2 m-1 .
$$

This is the Painlevé II equation, with a constant, and it also arises in the scaling solution of unitary matrix models with boundaries[9]. Its solutions have been studied in detail as well[10]. Note that in the present case, the constant appearing on the right hand side of (42) is an odd positive integer, while the constant in $[9,10]$ is an arbitrary integer corresponding to the number of quark flavors coupled to the models.

The above results are valid for any $m \geq 1$. From (5), one has $\phi_{0}^{(l)}=0$, so $g_{0}=0$. Thus at $m=1,(40)$ and (41) give: $0=g_{1}+\partial_{t} f_{1}$ and $0=t+g_{1}-f_{1}^{2}$. Therefore $f_{1}$ satisfies

$$
t=\partial_{t} f_{1}+f_{1}^{2}
$$

the $k=2$ scaling equation in the hierarchy arising from vector models[3]. This result is consistent with (42), which can be written as

$$
\left(\partial_{t}-2 f_{m}\right)\left(\partial_{t} f_{m}+f_{m}^{2}-t\right)=2(m-1)
$$

Hence at $m=1,(42)$ contains the second vector equation (43).

Since $M$ does not scale, the relation of $f_{m}$ to the free energy is slightly more complicated than in other matrix models. One has

$$
\exp \left(-\partial_{P} F_{M}\right) \approx \frac{\mathcal{Z}_{M, P+1}}{\mathcal{Z}_{M, P}}=\prod_{m=1}^{M} \frac{h_{m-1}^{(P+1)}}{h_{m-1}^{(P)}}=\prod_{m=1}^{M} \theta_{m}^{(P)}
$$

which leads to

$$
\partial_{t} F_{M}=-\sum_{m=1}^{M} f_{m}
$$

Given the asymptotic expansion arising from (42)

$$
f_{m}=t^{\frac{1}{2}}-\frac{2 m-1}{4} t^{-1}-\frac{12 m(m-1)+5}{32} t^{-\frac{5}{2}}-\ldots
$$

one finds

$$
\partial_{t} F_{M}=-M t^{\frac{1}{2}}+\frac{M^{2}}{4} t^{-1}+\frac{4 M^{3}+M}{32} t^{-\frac{5}{2}}+\ldots
$$

These results agree with those for the vector models using the analysis given in [3]. One finds, therefore, an a priori surprising connection between solutions of (42), and the Wronskian of the flows of Burger's equation[3]. 
Therefore we have confirmed that the scaling behavior of this critical point corresponds to a particular scaling solution of the vector models, which describe dynamically branched polymers. This case generalizes to the complete hierarchy of vector critical points, which are characterized by $\phi_{c}=0=q_{c}$. Without giving the full analysis, we give the solution for the next critical point in this series. When $g_{c}=1, q_{c}=0, \theta_{c}=1, \phi_{c}=0, a_{1}=-a_{2}=3, a_{3}=1$, we find $\mu=\nu=\frac{1}{4}, \hat{\mu}=\frac{1}{2}, \hat{\nu}=0$, and

$$
\begin{aligned}
& 0=m+g_{m}\left(f_{m}^{2}+f_{m} f_{m+1}+f_{m+1}^{2}-g_{m-1}-g_{m}-g_{m+1}\right) \\
& 0=t-f_{m}^{3}+f_{m-1} g_{m-1}+g_{m} f_{m+1}+2 f_{m}\left(g_{m}+g_{m-1}\right) .
\end{aligned}
$$

Eq. (40) remains unchanged, and combined with the above results, we find

$$
\begin{aligned}
0=t^{2} f_{m} & +6(1-2 m) f_{m}^{3}+\left(4 t-5 f_{m}^{3}\right) f_{m}^{4}-4 f_{m} \partial_{t} f_{m} \\
& +\left(4 t+5 f_{m}^{3}\right)\left(\partial_{t} f_{m}\right)^{2}-\left(4 t-10 f_{m}^{3}\right) f_{m} \partial_{t}^{2} f_{m}-4\left(\partial_{t} f_{m}\right)^{2} \partial_{t}^{2} f_{m} \\
& +3 f_{m}\left(\partial_{t}^{2} f_{m}\right)^{2}+4 f_{m} \partial_{t} f_{m} \partial_{t}^{3} f_{m}-2 f_{m}^{2} \partial_{t}^{4} f_{m} .
\end{aligned}
$$

One also finds that at $m=1$

$$
t=f_{1}^{3}+3 f_{1} \partial_{t} f_{1}+\partial_{t}^{2} f_{1}
$$

which is the $k=3$ vector scaling equation. There is also a topological (i.e., nonsingular) scaling solution for $g_{c}=1, q_{c}=0, \theta_{c}=1, \phi_{c}=0, a_{1}=1$, with $\mu=\nu=\frac{1}{2}, \hat{\mu}=1, \hat{\nu}=0$, and $g_{m}=-m, f_{m}=t$. In all cases, (44) determines the free energy given the scaling solutions for $f_{m}$.

(ii) $y=-1: \quad g_{c}=1, q_{c}=1, \theta_{c}=1, \phi_{c}=1, a_{1}=-2, a_{2}=1$

We find very similar scaling behavior at this critical point despite the fact that it describes very different physics. This critical point involves square matrices since $(P / \beta)_{c}=g_{c}-q_{c}=0$, though they may deviate from being precisely square in the scaling limit. Since $\theta_{c}-\phi_{c}=0$, (22) indicates that the critical behavior involves the approach of the inner limit of the eigenvalue distribution to the origin. For $P=0$ exactly, a series of scaling solutions for such critical points have been studied in Ref. 25 .

Considering the expansion about the critical point, one has $\hat{q}=q_{c}-g_{c}=-P / \beta$ and $\hat{\phi}=\phi+\theta$. A convenient choice of variables to complete the description of the singularity are $q$ and $\theta-\phi$. Defining $\Delta_{(+)} \equiv \Delta(\theta+\phi)$ and $\Delta_{(-)} \equiv \Delta(\theta-\phi),(36)$ becomes

$$
\Delta(g-q)=\Delta_{(+)} \Delta_{(-)}, \quad \Delta q=2 \Delta_{(+)}-\frac{1}{4} \Delta_{(-)}^{2}
$$

where we have dropped terms that are higher order in the scaling limit. Rewriting (45) as $\Delta(g-q)=\frac{1}{4} \Delta_{(-)}^{3}+\frac{1}{2} \Delta_{(-)} \Delta q$, one recognizes that this critical point is again characterized by a Whitney pleat[11].

We find a scaling solution similar to that for case (i). Defining $\delta \equiv \beta^{-\frac{1}{3}}$, we set

$$
\begin{aligned}
\frac{P}{\beta} & =\delta^{3} t \\
\frac{M}{\beta} & =1-\delta^{2} s,
\end{aligned}
$$


and

$$
\begin{aligned}
& \theta_{M+n}^{(l)}+\phi_{M+n}^{(l)}=2-2 \exp \left[-n \delta \partial_{s}\right]\left(\delta^{2} h_{2}^{(l)}(s)+\delta^{3} h_{3}^{(l)}(s)+\ldots\right) \\
& \theta_{M+n}^{(l)}-\phi_{M+n}^{(l)}=-2 \exp \left[-n \delta \partial_{s}\right]\left(\delta^{1} k_{1}^{(l)}(s)+\delta^{2} k_{2}^{(l)}(s)+\ldots\right) .
\end{aligned}
$$

Hence in this case, $P$ does not scale, so no derivatives with respect to $t$ are introduced. Eq.'s $(12,13)$ produce scaling solutions for fixed $l$. One finds

$$
\begin{aligned}
& h_{2}^{(l)}=\frac{1}{4}\left(s-k_{1}^{(l) 2}+\partial_{s} k_{1}^{(l)}\right), \\
& h_{3}^{(l)}=-\frac{1}{8}\left(l+4 k_{1}^{(l)} k_{2}^{(l)}-2 \partial_{s} k_{2}^{(l)}\right) .
\end{aligned}
$$

The scaling function $k_{1}^{(l)}$ satisfies

$$
\partial_{s}^{2} k_{1}^{(l)}-2 k_{1}^{(l) 3}+2 s k_{1}^{(l)}=2 l+1
$$

which is the Painlevé II equation with a constant, once again. The scaling parameters associated with $M$ and $P$ have traded rôles in (48), as compared to (42). Since $M / \beta$ scales in the present case, (33) allows one to determine the free energy in terms of $k_{1}^{(l)}$ as

$$
\partial_{s}^{2} F^{(P)}=-\frac{1}{2}\left(s+k_{1}^{(P)_{2}}+\partial_{s} k_{1}^{(P)}\right)
$$

For this critical point, the potential independent results derived from $(8,9)$ play an auxiliary rôle, relating the scaling function at different values of $l$

$$
k_{1}^{(l) 2}-\partial_{s} k_{1}^{(l)}=k_{1}^{(l+1) 2}+\partial_{s} k_{1}^{(l+1)} .
$$

Thus the free energies at different values of $P$ will also be related for these models.

The above results remain unchanged at $l$ or $P=0$. This corresponds to the case of precisely square matrices, which were studied in Ref. 25. As noted in case (i), one can write (48) with $l=0$ as

$$
\left(\partial_{s}-2 k_{1}^{(0)}\right)\left(\partial_{s} k_{1}^{(0)}+k_{1}^{(0)} 2-s\right)=0
$$

Thus the conventional solution of (48) with the asymptotic expansion $k_{1}^{(0)}= \pm s^{1 / 2}+\ldots$, leads to a trivial free energy $\partial_{s}^{2} F^{(0)}=-s$ with no higher order terms. Fortunately, the Painlevé II equation with a nonvanishing constant admits a third solution, which for $l=0$ is

$$
k_{1}^{(0)}=\frac{1}{2} s^{-1}-\frac{3}{8} s^{-4}+\frac{111}{32} s^{-7}-\ldots
$$

yielding

$$
\partial_{s}^{2} F^{(0)}=-\frac{1}{2} s+\frac{1}{8} s^{-2}-\frac{9}{16} s^{-5}+\frac{1323}{128} s^{-8}-\ldots .
$$

We have checked that up to rescaling variables, the universal (i.e., nonanalytic) part of the free energy is identical to that found for the first critical model in Ref. 25. Note that the 
latter analysis describes the free energy in terms of a scaling function, which satisfies Painlevé II without a constant.

This case generalizes to a hierarchy of critical points characterized by $\theta_{c}=\phi_{c}$ and $g_{c}=q_{c}$. At $P=0$, our analysis will reproduce the results of Ref. 25. There a new series of scaling solutions describing random surfaces, was constructed. Their asymptotic expansions remain governed by the conventional differential equations arising in the analysis of Hermitian matrix models[1]. For $P \neq 0$, the matrices in the present models, deviate slightly from being square. The scaling solutions of these matrix models are the limit II solutions discussed in [2]. The relation between the present analysis and that given in the previous references for both these random surface and the previous vector critical points is under investigation[18].

\section{Scaling Solutions with Partial Differential Equations}

We now return, for generic values of $y$, to (36) which gave the expansion about the critical points associated with the simplest nontrivial potential. Along with $\hat{\phi}=(\phi-y \theta)$ and $\hat{q}=(q+y g)$, we choose $\theta$ and $g$ to give a representative parameterization of the singularity, (36) becomes

$$
\begin{aligned}
& \Delta g=-\frac{2}{2 y+1} \Delta \hat{\phi}-\Delta \theta^{2}-\frac{2}{2 y+1} \Delta \hat{\phi} \Delta \theta \\
& \Delta \hat{q}=-2 \Delta \hat{\phi} \Delta \theta-\frac{1}{2 y+1}\left(\Delta \hat{\phi}^{2}+3 y(y+1) \Delta \theta^{2}\right) .
\end{aligned}
$$

Now as long as $y(y+1) \neq 0$, one can define $\hat{g}=g-(2 y+1) \hat{q} / 3 y(y+1)$ for which the relevant expansion is simply

$$
\Delta \hat{g}=-\frac{2}{2 y+1} \Delta \hat{\phi}
$$

since the remaining quadratic terms contain $\Delta \hat{\phi}$ and hence are higher order in the scaling limit. Note that (51) is the complete expansion. Cubic or higher order terms are simply not possible since the potential $V(y)$ is only quadratic. Choosing $\hat{\theta}=\theta+(2 y+1) \hat{\phi} / 3 y(y+1)$, the $\hat{q}$ expansion becomes

$$
\Delta \hat{q}=-\frac{1}{2 y+1}\left(3 y(y+1) \Delta \hat{\theta}^{2}-\frac{y^{2}+y+1}{3 y(y+1)} \Delta \hat{\phi}^{2}\right) .
$$

Eq.'s $(51,52)$ characterize the generic singularities. Clearly this analysis is inappropriate for $y=0$ and -1 , which verifies the claim in the previous section that these are higher order multicritical points where the form of the singularity changes.

The quadratic form of the singularity in (52), might lead one to conjecture that $\Delta \hat{q} \approx$ $-\delta^{4} t$ where $\delta=\beta^{-\frac{1}{5}}$. The exponents are chosen here in analogy to those which characterize the Painlevé I equation, which arises from a quadratic singularity in the standard doublescaling analysis. Given this choice, (51) would lead to $\Delta \hat{g} \approx-\delta^{2} s$. Discrete differences in the large- $N$ parameters, $\beta \hat{g}$ and $\beta \hat{q}$, would then give rise to derivatives, $\beta^{-\nu} \partial_{t}=\delta \partial_{t}$ and $\beta^{-\hat{\nu}} \partial_{s}=\delta^{3} \partial_{s}$, respectively. We will find that these choices do indeed lead to a triple-scaling 
solution below. An essential feature of this solution is the ratio $\hat{\nu} / \nu=3$. We shall show that with this choice, a partial differential equation very similar to the full time-dependent $\mathrm{KdV}$ equation is obtained from $(8,9)$, without any assumptions restricting $V(y)$. Suitable tunings of $V(y)$ lead to a hierarchy of additional differential equations. Each of these new critical points is governed by a system of two partial differential equations, one of which is the $\mathrm{KdV}$-like equation.

\subsection{Potential Independent Relations}

In accord with (26), we begin with a general scaling ansatz for two linearly independent combinations of $M$ and $P$

$$
\begin{aligned}
& \frac{A M+C P}{\beta}=\left[\frac{A M+C P}{\beta}\right]_{c}-\beta^{\nu-1} t \\
& \frac{B M+D P}{\beta}=\left[\frac{B M+D P}{\beta}\right]_{c}-\beta^{\hat{\nu}-1} s .
\end{aligned}
$$

In the scaling limit, discrete differences become derivatives as follows:

$$
\partial_{M}=-\beta^{-\nu} A \partial_{t}-\beta^{-\hat{\nu}} B \partial_{s} \quad \text { and } \quad \partial_{P}=-\beta^{-\nu} C \partial_{t}-\beta^{-\hat{\nu}} D \partial_{s} .
$$

Now set $\hat{\nu} / \nu=3$ as above, and define $\delta \equiv \beta^{-\nu}$. We also choose $\theta_{c}=1$ and $\phi_{c}=y^{2}$ as in previous sections, and then introduce

$$
\begin{aligned}
\theta_{M+n}^{(P+l)} & =1-\exp \left[-\delta(A n+C l) \partial_{t}-\delta^{3}(B n+D l) \partial_{s}\right] \sum_{q=2} \delta^{q} h_{q}(t, s) \\
\phi_{M+n}^{(P+l)} & =y^{2}-\exp \left[-\delta(A n+C l) \partial_{t}-\delta^{3}(B n+D l) \partial_{s}\right] \sum_{q=2} \delta^{q} k_{q}(t, s)
\end{aligned}
$$

Substituting (55) into $(8,9)$, we find

$$
y= \pm \frac{C}{A-C} .
$$

One may choose the plus sign here without any loss of generality. Then

$$
\begin{aligned}
& k_{2}(t, s)=y\left(h_{2}(t, s)+g_{2}(s)\right) \\
& k_{3}(t, s)=y\left(h_{3}(t, s)-\frac{A}{2} \partial_{t} h_{2}(t, s)+g_{3}(s)\right),
\end{aligned}
$$

and finally, one solves for $k_{4}$ at $O\left(\delta^{5}\right)$, but consistency between (8) and (9) requires

$$
\partial_{s} h_{2}=\frac{C^{2}}{4 T} \frac{y-1}{y^{2}} \partial_{t}\left[h_{2}^{2}-\frac{C^{2}}{6} \frac{y+1}{y} \partial_{t}^{2} h_{2}\right]-\frac{C^{2}}{2 T y^{2}} g_{2} \partial_{t} h_{2}-\frac{1}{2} \partial_{s} g_{2}
$$

where $T=A D-B C . T$ is the determinant of the linear transformation relating $\Delta(M / \beta)$ and $\Delta(P / \beta)$ to the scaling variables, $t$ and $s$, in (53), so we require that $T \neq 0$. Neglecting 
the last two terms involving $g_{2},(58)$ is the $\mathrm{KdV}$ equation with $s$ and $t$ playing the rôles of the time and space coordinates, respectively. We stress that this result was obtained only assuming a particular ratio of the exponents in (53) and (55) (i.e., $\hat{\nu} / \nu=3$ and $q \geq 2$ ), without restricting the matrix potential in any way.

\subsection{Potential Dependent Results for Quadratic Potential}

We return to discussing the generic critical point associated with the quadratic potential. For a scaling solution of $(12,13)$, one finds $\nu=\frac{1}{5}$,

$$
g_{2}(s)=-\frac{C}{2 T} \frac{2 y+1}{y^{2}} s \quad \text { and } \quad g_{3}(s)=0
$$

Finally $h_{2}$ must satisfy the following differential equation

$$
0=t-\frac{C y}{2 y+1} g_{2}^{2}-2 C g_{2} h_{2}-3 C \frac{y+1}{2 y+1}\left[h_{2}^{2}-\frac{C^{2}}{6} \frac{y+1}{y} \partial_{t}^{2} h_{2}\right]
$$

This equation has a form similar to the Painlevé I equation, again up to terms involving $g_{2}$, which introduce $s$ dependence.

Up until this point, we have not made use of the freedom available to rescale the variables: $s \rightarrow \varpi s$ and $t \rightarrow \varphi t$; and the functions: $h_{2} \rightarrow \gamma h_{2}$ and $g_{2} \rightarrow \eta g_{2}$; to simplify our results. Note that these rescalings do not provide enough freedom to completely eliminate $y$ from the coefficients in $(58,59,60)$. A suitable rescaling brings these equations to the following form: $g_{2}=s$

$$
\begin{aligned}
0 & =t-\frac{3 y(y+1)}{y^{2}+y+1} g_{2}{ }^{2}+2 \frac{2 y+1}{\sqrt{y^{2}+y+1}} g_{2} h_{2}-h_{2}{ }^{2}+\frac{9}{2} \partial_{t}^{2} h_{2} \\
\partial_{s} h_{2} & =\frac{y-1}{2 \sqrt{y^{2}+y+1}} \partial_{t}\left[h_{2}^{2}-\frac{9}{2} \partial_{t}^{2} h_{2}\right]+\frac{3(y+1)}{y^{2}+y+1} g_{2} \partial_{t} h_{2}+\frac{3(y+1)}{2 \sqrt{y^{2}+y+1}} \partial_{s} g_{2} .
\end{aligned}
$$

Although $C$ and $T$ have been eliminated from the coefficients, the last two equations still appear rather complicated. The true simplicity of our results is made manifest by applying $g_{2}=s$, and shifting $h_{2}=\hat{h}+(2 y+1) s / \sqrt{y^{2}+y+1}$, which then yields

$$
\begin{aligned}
& 0=\left(t+s^{2}\right)-\hat{h}^{2}+\frac{9}{2} \partial_{t}^{2} \hat{h} \\
& 0=\partial_{s} \hat{h}-2 s \partial_{t} \hat{h}
\end{aligned}
$$

where $\partial_{t}^{3} \hat{h}$ has also been eliminated from the KdV-like equation, by using the first equation above. Thus (61) shows that all values of $y$ (which indirectly determines the proportions of the matrices) give the same scaling behavior. Note though that the shift between $h_{2}$ and $\hat{h}$ introduces a separate $s$ dependence with a coefficient involving $y$. 
Given the scaling equations (61), there are some complications in determining the free energy. Eq. (54) shows that both $\partial_{M}$ and $\partial_{P}$ are both proportional to $\partial_{t}$, to leading order. From (33), the expression for $\alpha_{M}^{(P)}$ yields

$$
\partial_{t}^{2} F \approx-\frac{1}{\delta^{2}} \frac{2 y^{2} \log y}{C^{2}(y+1)^{2}}+\frac{y}{C^{2}(y+1)} h_{2}+\frac{y}{C^{2}(y+1)^{2}} g_{2}
$$

while that for $\theta_{M}^{(P)}$ yields

$$
\partial_{t}^{2} F \approx \frac{y}{C^{2}(y+1)} h_{2}
$$

The disagreement between these expressions arises because the different approximations introduce different nonuniversal contributions. The divergent constant explicitly included in (62) is a familiar term, which is regularly dropped (as we did implicitly in section 3). The finite difference between (62) and (63) proportional to $g_{2}(s)$ may be less familiar, but is simply another nonuniversal contribution. Consistent expressions for $\partial_{M} \partial_{P}^{2} F, \partial_{M}^{2} \partial_{P} F$ and $\partial_{M}^{3} F$ can be produced by taking appropriate ratios of the recursion coefficients $\theta$ and $\alpha$, with the result:

$$
\partial_{t}^{3} F \approx \frac{y}{C^{2}(y+1)} \partial_{t} h_{2}
$$

Therefore up to rescalings which can be absorbed in $F$, we define the universal part of the free energy as

$$
F(t, s)=\int^{t} d t_{1} \int^{t_{1}} d t_{2} \hat{h}\left(t_{2}, s\right)
$$

where any possible analytic dependence on $t$ is dropped, as well as any separate $s$ dependence. ${ }^{\dagger}$

Now consider solving the scaling equations (61). The second equation is simply solved by $\hat{h}=\hat{h}\left(t+s^{2}\right)$. Given this form, the first equation can be solved by a perturbative expansion identical to that constructed for the Painlevé I equation, where the expansion parameter is

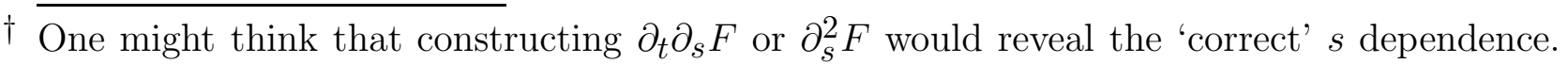
We were unable to find consistent definitions in general. For instance, one might expect that $\partial_{t} \partial_{s} F$ will appear to leading order in $\partial_{M}\left(C \partial_{M}-A \partial_{P}\right) F$, but either $\log \left[\left(\alpha_{M}^{(P)}\right)^{C} /\left(\theta_{M}^{(P)}\right)^{A}\right]$ or $\log \left[\left(\alpha_{M}^{(P)}\right)^{C} /\left(\theta_{M+1}^{(P)}\right)^{A}\right]$ appear to be equally good approximations for this quantity. Unfortunately the two expressions differ at the subleading order $\delta^{3}$, while $\partial_{t} \partial_{s} F$ appears at order $\delta^{4}$. 
now $\left(t+s^{2}\right)^{-5 / 2}$. Thus one finds the free energy is given by

$$
\begin{aligned}
F= & \frac{4}{15}\left(t+s^{2}\right)^{\frac{5}{2}}+\frac{9}{16} \ln \left(t+s^{2}\right)-\frac{567}{640}\left(t+s^{2}\right)^{-\frac{5}{2}}+\ldots \\
= & \frac{4}{15} t^{\frac{5}{2}}\left(1+\frac{5}{2} \frac{s^{2}}{t}+\frac{15}{8} \frac{s^{4}}{t^{2}}+\ldots\right) \\
& +\frac{9}{16} \ln (t)+\left(\frac{9}{16} \frac{s^{2}}{t}-\frac{9}{32} \frac{s^{4}}{t^{2}}+\ldots\right) \\
& -\frac{567}{640} t^{-\frac{5}{2}}\left(1-\frac{5}{2} \frac{s^{2}}{t}+\frac{35}{8} \frac{s^{4}}{t^{2}}+\ldots\right)+\ldots
\end{aligned}
$$

These results can be interpreted as follows: The free energy has the conventional topological expansion in terms of $t^{-\frac{5}{2}}$, but the contribution of each topology is perturbed by terms $\left(s^{2} / t\right)^{m}=\left(s / t^{\frac{1}{2}}\right)^{2 m}$. In the present case, these perturbations can be resummed to give an overall renormalization of the topological expansion parameter of the form $\left(t+s^{2}\right)^{-\frac{5}{2}}$.

It is obvious from (65)that there is a dual asymptotic solution, valid when $s$ is large and $t$ is small. This dual expansion merely has $t^{\frac{5}{2}}$ replaced with $s^{5}$, with $s^{2} / t \rightarrow t / s^{2}$. In the next section, we shall find that the dual expansion has a rather distinct character-neverthelsss, the important point is the existence of a dual expansion for these critical points.

\subsection{Higher Order Singularities}

This section discusses some results for the higher order multicritical models, for which the KdV-like equation governs the scaling behavior. In section 3, we described how a matrix potential with $(2 n-1)$ nonvanishing coefficients could be tuned to to yield a split singularity of the form $y \Delta g \pm \Delta q \propto(y \Delta \theta \pm \Delta \phi)^{n}$. One extra nonvanishing coefficient (for a total of $2 n$ ) will allow for extra tuning so that $y \Delta g+\Delta q$ can be made to vanish to order $n$ in the variations, $\Delta \theta$ and $\Delta \phi .^{\ddagger}$ This construction leads to the singularities where the scaling solution of section 5.1 is relevant.

Explicitly for the case $n=2$, the minimal potential has $a_{1}, a_{2}, a_{3}$ and $a_{4}$ nonvanishing. We choose $g_{c}=1=\theta_{c}$ and $\phi_{c}=y^{2}$. Then setting

$$
\begin{aligned}
& a_{1}=-2\left(2 y^{6}+3 y^{5}+3 y+2\right) a_{4} \\
& a_{2}=6\left(y^{4}+y^{3}+y^{2}+y+1\right) a_{4} \\
& a_{3}=-2\left(2 y^{2}+y+2\right) a_{4} \\
& a_{4}=\frac{1}{6 y^{3}+2 y^{2}-2 y-1}
\end{aligned}
$$

yields a singularity of the form

$$
\begin{aligned}
& \Delta g \propto \Delta \hat{\phi}^{2} \\
& \Delta \hat{q} \propto b_{3} \Delta \hat{\phi}^{3}+b_{2} \Delta \hat{\phi}^{2} \Delta \theta+b_{1} \Delta \hat{\phi} \Delta \theta^{2}+\Delta \theta^{3}
\end{aligned}
$$

$\ddagger$ Clearly we could have also singled out $y \Delta g-\Delta q$, but this yields the same results. 
where the notation is the same as used in (50), and $b_{i}$ are constants depending on $y$. Note that in this case unlike (52), one cannot choose a linear combination $\hat{\theta}=\theta+x \hat{\phi}$ to eliminate all of the terms mixing $\Delta \hat{\phi}$ and $\Delta \hat{\theta}$ in the singularity, although the term linear in $\Delta \hat{\phi}$ could be removed. For (66), one finds that $q_{c}=y^{5}\left(6+2 y-2 y^{2}-y^{3}\right) /\left(6 y^{3}+2 y^{2}-2 y-1\right)$. Requiring $g_{c}=1>q_{c}>0$, leaves two possible ranges for $y$ : $-1<y<0$ or $1<y<\xi$ with $\xi=-2 / 3+10^{1 / 3} / 3+100^{1 / 3} / 3 \approx 1.59867$.

Now one may investigate a scaling solution with the ansatz given in section 5.1. The results presented there $(56,57,58)$, are of course unchanged. A scaling solution of $(12,13)$ requires

$$
\begin{aligned}
\nu= & \frac{1}{7} ; \quad g_{2}=s^{\frac{1}{2}} ; \quad g_{3}=0 ; \\
0= & t-h_{2}^{3}+h_{2} \partial_{t}^{2} h_{2}+\frac{1}{2}\left(\partial_{t} h_{2}\right)^{2}-\frac{1}{10} \partial_{t}^{4} h_{2} \\
& +\frac{1}{2} g_{2} \partial_{t}^{2} h_{2}-\frac{3}{2} g_{2} h_{2}^{2}-\frac{3}{5} \frac{y^{2}+3 y+1}{(y+1)^{2}} g_{2}{ }^{2} h_{2}-\frac{1}{10} \frac{y(y+4)}{(y+1)^{2}} g_{2}{ }^{3}
\end{aligned}
$$

where rescalings have been made to simplify these results. A further simplification comes from shifting $h_{2}=\hat{h}-\frac{1}{2} g_{2}$, applying $g_{2}=s^{\frac{1}{2}}$, and making the following definitions:

$$
x=\frac{y-1}{y+1}, \quad \tau=t-\frac{x}{20} s^{\frac{3}{2}}, \quad \sigma=\frac{3 x^{2}}{20} s,
$$

which yields

$$
0=\tau-\hat{h}^{3}+\hat{h} \partial_{\tau}^{2} \hat{h}+\frac{1}{2}\left(\partial_{\tau} \hat{h}\right)^{2}-\frac{1}{10} \partial_{\tau}^{4} \hat{h}+\sigma \hat{h} .
$$

Neglecting the last term involving $\sigma$, this is the second equation in the hierarchy that arises for scaling solutions of hermitian matrix models[1]. With the corresponding rescalings and the shift of $h_{2},(58)$ becomes the $\mathrm{KdV}$ equation

$$
\partial_{\sigma} \hat{h}=\frac{1}{2} \partial_{\tau}\left[\hat{h}^{2}-\frac{1}{3} \partial_{\tau}^{2} \hat{h}\right] .
$$

Note that all of the $y$ dependence has been absorbed in the definitions of $\tau$ and $\sigma$, leaving the scaling behavior independent of the proportions of the matrices, once again.

One can construct a perturbative solution of these scaling equations for large $\tau$ :

$$
\begin{aligned}
F= & \frac{9}{28} \tau^{\frac{7}{3}}\left[1+\frac{14}{15} \frac{\sigma}{\tau^{\frac{2}{3}}}+\frac{14}{81} \frac{\sigma^{3}}{\tau^{2}}+\frac{7}{243} \frac{\sigma^{4}}{\tau^{\frac{8}{3}}}+\ldots\right] \\
& +\frac{1}{18} \ln \tau+\left[\frac{1}{36} \frac{\sigma}{\tau^{\frac{2}{3}}}+\frac{1}{216} \frac{\sigma^{2}}{\tau^{\frac{4}{3}}}-\frac{1}{243} \frac{\sigma^{3}}{\tau^{2}}+\frac{11}{11664} \frac{\sigma^{4}}{\tau^{\frac{8}{3}}}+\ldots\right] \\
& -\frac{1}{120} \tau^{-\frac{7}{3}}\left[1+\frac{25}{81} \frac{\sigma}{\tau^{\frac{2}{3}}}-\frac{170}{243} \frac{\sigma^{2}}{\tau^{\frac{4}{3}}}+\frac{505}{729} \frac{\sigma^{3}}{\tau^{2}}+\ldots\right]+\ldots
\end{aligned}
$$

These results are more complicated than the previous case, (65): a conventional topological expansion can be constructed in terms of $t^{-\frac{7}{3}}$, dressed at each order by perturbations 
$\left(s^{\frac{1}{2}} / t^{\frac{1}{3}}\right)^{m}$. The odd powers can be absorbed in an overall renormalization of the expansion parameter, $t \rightarrow \tau$, as seen explicitly in (69). Perturbations with even powers remain in (69), and can not be incorporated into such a renormalization. In part, the latter complication arises because eq. (68) cannot be reduced to a linear equation as in (61). Perhaps then one should think of (61) as the 'topological point' for the physics described by these partial differential equations.

The dual expansion at this critical point $\left(\right.$ for $\left.\sigma^{3} \gg \tau^{2}\right)$ is

$$
\begin{aligned}
\hat{h}= & \sigma^{\frac{1}{2}}\left[1+\frac{1}{2} \frac{\tau}{\sigma^{\frac{3}{2}}}-\frac{3}{8} \frac{\tau^{2}}{\sigma^{3}}+\frac{1}{2} \frac{\tau^{3}}{\sigma^{\frac{9}{2}}}-\frac{105}{128} \frac{\tau^{4}}{\sigma^{6}}+\ldots\right] \\
& -\frac{5}{16} \sigma^{-3}\left[1-\frac{51}{10} \frac{\tau}{\sigma^{\frac{3}{2}}}+\frac{393}{20} \frac{\tau^{2}}{\sigma^{3}}-\frac{5403}{80} \frac{\tau^{3}}{\sigma^{\frac{9}{2}}}+\frac{3489}{16} \frac{\tau^{4}}{\sigma^{6}}+\ldots\right] \\
& -\frac{2451}{512} \sigma^{-\frac{13}{2}}\left[1-\frac{9406}{817} \frac{\tau}{\sigma^{\frac{3}{2}}}+\frac{531501}{6536} \frac{\tau^{2}}{\sigma^{3}}-\frac{741305}{1634} \frac{\tau^{3}}{\sigma^{\frac{9}{2}}}+\frac{229444887}{104576} \frac{\tau^{4}}{\sigma^{6}}+\ldots\right]+\ldots
\end{aligned}
$$

Again, note that it is not possible to absorb the perturbations, now in powers of $\tau \sigma^{-\frac{3}{2}}$, into a renormalization of $\sigma$. There is an analogous series with $\hat{h} \sim-\sigma^{\frac{1}{2}}+\ldots$ We have given here the expansion of the specific heat, not the free energy, because our methods (as mentioned in a preceding footnote) do not enable us to unambiguously determine the derivatives of the free energy with respect to $s$. Thus, we are not able to determine from the triple-scaling equations, contributions which are non-analytic in $s$ but have a $\tau$ dependence of the form $a+b \tau$. It would be a tedious but straightforward exercise to obtain these terms using the techniques of [26]. Note that the dual expansions $(69,70)$ are in terms of the renormalized parameters $\tau, \sigma$. Similar expansions in terms of the bare parameters $t, s$ can be constructed, as in (65).

There are instanton solutions associated with each of these weak-coupling expansions. Linearizing $(67,68)$ about $(69)$, we find solutions of the form

$$
\varepsilon(\sigma, \tau)=\tau^{-\frac{1}{4}}\left(1+a_{1} \frac{\sigma}{\tau^{\frac{2}{3}}}+\ldots\right) \exp \left[-\frac{6}{7} \tau^{\frac{7}{6}}\left\{r_{0}+r_{1} \frac{\sigma}{\tau^{\frac{2}{3}}}+\ldots\right\}\right]
$$

where $r_{0}: 30-10 r_{0}^{2}+r_{0}^{4}=0, r_{1}=-7 r_{0}\left(r_{0}^{2}-6\right) / 36$, and $a_{1}=\left(r_{0}^{2}+2\right) / 24$. At $\sigma=0$, these solutions reduce to those found for the $k=3$ Hermitian matrix model[1].

Similarly, linearizing about (70), we find solutions of the form

$$
\epsilon(\sigma, \tau)=\sigma^{-\frac{3}{8}}\left(1+a_{1} \frac{\tau}{\sigma^{\frac{3}{2}}}+\ldots\right) \exp \left[-\frac{4}{7} \sigma^{\frac{7}{4}}\left\{r_{0}+r_{1} \frac{\tau}{\sigma^{\frac{3}{2}}}+\ldots\right\}\right]
$$

where now $r_{1}: 12005-1960 r_{1}^{2}+64 r_{1}^{4}, r_{0}=4 r_{1}\left(147-8 r_{1}^{2}\right) / 1029$, and $a_{1}=r_{1}^{2} / 49-1 / 4$. Note that in both instances, the prefactor and the exponential are 'dressed' by series in the same perturbing parameters as $(69,70)$. We have exhibited instantons for this critical point to display this 'dressing'. The instantons for the other critical points found in this paper do not differ from the double-scaling analysis instantons. 
Analogous behaviour is found for higher multicritical points in this series. For general $n$, the singularity may be put in the form

$$
\begin{aligned}
& \Delta g \propto \Delta \hat{\phi}^{n} \\
& \Delta \hat{q} \propto \Delta \hat{\theta}^{n+1}+b_{2} \Delta \hat{\phi}^{2} \Delta \hat{\theta}^{n-1}+\ldots
\end{aligned}
$$

where '...' indicates terms higher order in $\Delta \hat{\phi}$. In the scaling solution, $g_{2}=s^{\frac{1}{n}}$ while $\hat{h}$ satisfies a nonlinear equation similar to the $k=n+1$ equation in the usual hermitian matrix model hierarchy. This equation will yield the conventional topological expansion in $t^{-2-\frac{1}{n+1}}$, but perturbed by terms of the form $\left(s^{\frac{1}{n}} / t^{\frac{1}{n+1}}\right)^{m}$, as is evident by examining the planar limit. Finally, these perturbations cannot be expressed as a simple renormalization of the topological expansion parameter.

\section{Conclusions}

It is usually believed that the character of the large- $N$ limit is different for theories with dynamical variables transforming in vector-like represenations, or in the adjoint representation. The triple-scaling analysis given in the present work shows clearly that, at least for $d=0$ models, this perceived difference is a reflection of the limitations of a case-by-case analysis. We have shown explicitly that critical phenomena ranging from polymers to random surfaces are described by the same equations. Random surfaces are geometrically associated with triangulations defined by dual graphs of Feynman diagrams[15], while polymers are directly associated with the Feynman graphs[3]. One intriguing result is the appearance of the second Painlevé equation in a model of polymers. For $k$ even, one might expect that the higher equations should be related to the higher $\mathrm{mKdV}$ flows found in unitary matrix models - this will be discussed elsewhere[18].

We have endeavoured, in the present work, to provide a detailed account of the various subtleties that arise in the triple-scaling analysis. The methods given in this paper seem inappropriate for understanding the relation, if any, of the multicritical points of triplescaling models to hierarchies of integrable equations e.g., à la Drin'feld-Sokolov. Why should one expect such a relation? Firstly, the systems of equations obtained have a quasi-onedimensional character. Secondly, a detailed examination of the examples solved in this paper will lead to an appreciation of the miraculous cancellations that take place in order for scaling behaviour in both variables to occur - thus, integrability of some sort must underlie the triplescaling physics. The correct mathematical framework should, presumably, encompass the coupled differential equation-difference equation systems we found in sect. 4.

We found remarkable dual weak-coupling expansions for the examples in sect. 5. The transition from the domain of convergence of the sub-series associated with one expansion, to the domain of convergence of the 'dual' expansion, is of great physical interest - to understand this, note that small $s$ in the large $t$ expansion, (69), corresponds to strong coupling in the coupling constant associated with $s$, and vice versa in (70). Of course, it is necessary 
to solve the problem of the surface interpretation of the continuum theories before such a dual relationship can be exploited effectively.

We now suggest an interpretation of the physics of the new 'coupling constant' associated with the $s$ variable. The Liouville mode is the dynamical degree of freedom of induced two-dimensional gravity in background gauge. It is naturally associated with the string coupling constant, because of the linear dilaton-like[27] coupling of the Liouville field to the background curvature. In light of eq. (70), it is natural to conjecture the existence of a new dynamical field associated with the new expansion parameter. What might the physics of this new field be? Based on the surface interpretations that are suggested by finite $M, N$ computations[2,3], the new dynamics that can be investigated in the triple-scaling analysis would appear to involve microscopic topology fluctuations.

How might this happen? The saddle point analysis[20] computes the physics of random surfaces of spherical topology. Double-scaling[1] solves the physics of random surfaces to all orders in the topological expansion. The finer analysis provided by triple-scaling may then reveal more intricate sub-structure in the physics of random surfaces.

So much for that [28].

Appendix A. Another Higher Order Singularity

At the end of section 3, we mentioned that when the split singularity degenerates at $y^{2}=1$, the scaling solution is similar to that found in section 5 . We will briefly outline these results. With $y^{2}=1$, one has $g_{c}=1=q_{c}=\theta_{c}=\phi_{c}$ along with $a_{1}=-3, a_{2}=3, a_{3}=-\frac{1}{2}$. Defining $\Delta_{(+)} \equiv \Delta(\theta+\phi)$ and $\Delta_{(-)} \equiv \Delta(\theta-\phi)$, the singularity may be written as

$$
\Delta(g-q)=\frac{1}{4} \Delta_{(-)}^{3}-\frac{3}{4} \Delta_{(+)}^{2} \Delta_{(-)}, \quad \Delta(g+q)=-3 \Delta_{(+)}^{2}+\frac{3}{4} \Delta_{(+)} \Delta_{(-)}^{2} .
$$

The second term in $\Delta(g-q)$ is actually higher order in the scaling limit, but we explicitly retain it for comparison purposes below. We use the scaling ansatz presented in section 5.1, but the series in (55) is modified so that $q \geq 1$. We also fix $A=2, B=0, C=1$, and $D=1$ in $(53)$, and set $\hat{\nu} / \nu=2$. One finds that $\nu=\frac{1}{5}, k_{1}=-h_{1}=-(s / 2)^{\frac{1}{3}}$, and $k_{2}=h_{2}$ where $h_{2}$ satisfies

$$
\begin{aligned}
0 & =t-6 h_{1}^{2} h_{2}-12 h_{2}^{2}+4 \partial_{t}^{2} h_{2} \\
2 \partial_{s} h_{2} & =h_{1} \partial_{t} h_{2}-h_{1} \partial_{s} h_{1} .
\end{aligned}
$$

After suitable rescalings and a shift of $h_{2}$, one finds a scaling function $\hat{h}=\hat{h}\left(t+s^{\frac{4}{3}}\right)$, which satisfies the Painlevé I equation. This single function determines the universal part of the free energy as in (64). So the final result is that the free energy is identical to that found for pure gravity, except with a renormalized topological expansion parameter, $\left(t+s^{\frac{4}{3}}\right)^{-\frac{5}{2}}$.

We have been unable to generalize this scaling solution to higher order singularities. While the higher order split singularities all degenerate at $\phi_{c}=y^{2}=1$, for $n>2$ the singularities take the form

$$
\Delta(g-q) \propto \Delta_{(+)}^{n} \Delta_{(-)}, \quad \Delta(g+q) \propto \Delta_{(+)}^{n}+b \Delta_{(+)}^{n-1} \Delta_{(-)}^{2}
$$


where $b$ is some constant. These singularities all yield parametric scaling solutions for square matrices, as described in section 4 . Eq. (71) would have the same form with $n=2$, if it were

not for the appearance of the $\Delta_{(-)}^{3}$ term in $\Delta(g-q)$. Thus from this point of view, (71) seems to be an anomalous case.

We are grateful to Joe Minahan and C. Nappi for valuable communications. Some of the results in this paper were obtained (i) over two summers at the Aspen Center for Physics, (ii) while R.C.M. was visiting the Institute for Theoretical Physics at U.C.S.B. and (iii) while V.P. was visiting the Mathematical Sciences Research Insitute in Berkeley - we thank these institutions for their hospitality. This research was supported by NSERC of Canada, and Fonds FCAR du Québec (R.C.M.), by the NSF under Grant No. PHY89-04035, at UCSB, by the NSF Grant No. DMS85-05550 at M.S.R.I. (V.P.) and by DOE Grant No. DE-FG02-90-ER40542 at I.A.S. (V.P.)

\section{REFERENCES}

1. E. Brézin and V.A. Kazakov, Phys. Lett. B236 (1990) 144; M.R. Douglas and S.H. Shenker, Nucl. Phys. B335 (1990) 635; D.J. Gross and A.A. Migdal, Phys. Rev. Lett. 64 (1990) 127

2. A. Anderson, R.C. Myers and V. Periwal, Phys. Lett. 254B (1991) 89

3. A. Anderson, R.C. Myers and V. Periwal, Nucl. Phys. B360 (1991) 463

4. S. Nishigaki and T. Yoneya, Nucl. Phys. B348 (1991) 787

5. P. Di Vecchia, M. Kato and N. Ohta, Nucl. Phys. B357 (1991) 495

6. T.R. Morris, Nucl. Phys. B356 (1991) 703

7. R.C. Myers, McGill/ITP preprint NSF-ITP-91-69/McGill/91-17, to appear in Nuclear Physics B

8. G.M. Cicuta and E. Montaldi, Phys. Rev. D29 (1984) 1267; A. Barbieri, G.M. Cicuta and E. Montaldi, Nuo. Cim. 84A (1984) 173; C.M. Canali, G.M. Cicuta, L. Molinari and E. Montaldi, Nucl. Phys. B265 (1986) 485; G.M. Cicuta, L. Molinari, E. Montaldi and F. Riva, J. Math. Phys. 28 (1987) 1716

9. J.A. Minahan, Phys. Lett. 268B (1991) 29

10. J.A. Minahan, Phys. Lett. 265B (1991) 362; University of Virginia preprint UVA-HET91-07 (1991)

11. See for example: V.I. Arnold, S.M. Gusein-Zade and A.N. Varchenko, Singularities of Differentiable Maps: Volume I, Birkhäuser (Boston, 1985) 
12. R.C. Myers and V. Periwal, Phys. Rev. Lett. 64 (1990) 3111

13. E. Brézin and H. Neuberger, Phys. Rev. Lett. 65 (1990) 2098; Nucl. Phys. B350 (1991) 513

14. R.C. Myers and V. Periwal, Phys. Rev. Lett. 65 (1990) 1088

15. G. 't Hooft, Nucl. Phys. B72 (1974) 461

16. T.S. Chihara, Introduction to orthogonal polynomials, Gordon and Breach (New York, 1978)

17. D.J. Gross and A.A. Migdal, Nucl. Phys. B340 (1990) 333

18. R. Lafrance and R.C. Myers, in preparation

19. S. Dalley, C. Johnson, and T. Morris, 'Multicritical complex matrix models and nonperturbative 2-d quantum gravity', Southampton preprint SHEP-90-91-16 (1991)

20. E. Brézin, C. Itzykson, G. Parisi, and J.-B. Zuber, Comm. Math. Phys. 59 (1978) 35

21. See for example: N.I. Muskhelishvili, Singular Integral Equations, P. Noordhoff N.V. (Groningen, Holland, 1953)

22. C. Bachas and P.M.S. Petropolous, Phys. Lett. 247B (1990) 363

23. S. Dalley, 'Critical conditions for matrix models of string theory', Southampton preprint SHEP-90-91-6 (1990)

24. M.R. Douglas, N. Seiberg and S.H. Shenker, Phys. Lett. 244B (1990) 381

25. S. Dalley, C. Johnson and T. Morris, 'Non-perturbative two-dimensional quantum gravity', Southampton preprint SHEP-90-91-28 (1991)

26. D. Bessis, C. Itzykson, and J.-B. Zuber, Adv. Appl. Math. 1 (1980) 109

27. R.C. Myers, Phys. Lett. 199B (1987) 371

28. G. Stein, Brim Beauvais, in The Yale Gertrude Stein (ed. R. Kostelanetz), Yale University Press (New Haven, 1980) 